\title{
A Blind and Non-Blind Deblurring: An Algorithm for Deblurring based on Residual Whiteness in Images
}

\author{
M.Sahithya \\ M.Tech (DECS) Student \\ Dept.of E.C.E \\ AITS, Tirupati, India
}

\author{
I.Suneetha \\ Associate Professor\&Head \\ Dept.of E.C.E \\ AITS, Tirupati, India
}

\author{
N.Pushpalatha \\ Assistant Professor \\ Dept.of E.C.E \\ AITS, Tirupati, India
}

\begin{abstract}
Now a day's deblurring plays a crucial problem in many situations research due to the digital devices popularity such as digital camera, smart phone with camera etc. Aim of the image deblurring is making pictures sharp and useful. In previous methods do not find the perfect solution some disturbances are spectrally white occur in the image deblurring techniques. But In the proposed method compared to the non-blind deblur blind deblur gives the better results for synthetic and real life degradations with and without noise both in single and multiframe scenarios and also evaluate the whiteness in the image in terms of speed and restoration quality to compare the other deblurring techniques this paper yields better results .
\end{abstract}

\section{General Terms}

Blind and Non-Blind Deblurring.

\section{Key words}

Image deblurring, blind and Non-Blind deblurring, whiteness in the image

\section{INTRODUCTION}

In 1960s, the cold war between the United States and the Soviet Union at the time gave birth to the field of image deblurring. At that time peoples are main concentrate to producing pictures in the earth and the solar system. They need essential and important information from astronomical images so essentially need for the systematic image restoration techniques were brought to the engineering community. After done the many algorithms extensively increase the need for the astronomy and many others so to found their way called digital image deblurring. Generally images are classified into two types are constrained domain and unconstrained domain images. There is no disturbance of light such images are referred as constrained domain images and some images are adjusted before hand to facilitate use and there can be disturbance of light and posing problems these images are referred as unconstrained domain images. By using camera to record the image but every image has more or less blur is occur due to a lot of interference in camera or in the environment. Image deblurring is the general framework is use to convert the measurements of the observed image into information about a physical object or system where the observed image is to make as the convolution of a applications in image deblurring are medical imaging, photography, surveillance. Mainly Image deblurring is classified into two types are blind and non-blind. If the blurring kernel is to be known, is referred as non-blind deblurring where as the both the image and the blur kernel are unknown is referred as blind de-blurring. Non-blind Methods are much narrower than the blind methods: In most cases the response of an imaging system to a point source is referred as the point spread function is not known with good accuracy. So non blind methods are very sensitive to mismatches between the PSF is used by the method and the true blurring PSF, a poor knowledge of the blurring PSF normally leads to poor deblurring. In ID problems, the degraded image is designed as $y=h * x+n$ Where ' $y$ ' is degraded image, ' $x$ ' is original image, ' $\mathrm{x}$ ' is noise and $\mathrm{h}$ is the point spread function of the blur operator. In BID to finding ' $x$ ' and ' $h$ 'from ' $y$ ' and in NBID to find ' $x$ 'from ' $y$ ' and ' $h$ ' After many research has been (still is) devoted the blur operator in NBID is ill conditioned nature. So there is a slightly mismatch between the assumed blur and true one. so to overcome this problem by using an image regularize, or prior, the weight of which has to be tuned [1], [2] and the ratio of underlying wavelet/frame-based methods [3], [4]. In BID the blur operator is not ill-conditioned nature would still inherently illposed. Most BID methods limit the class of blur filters, either in a hard way or soft way .using parametric models in hard way [5],[6] and use of priors/regularizes in soft way[7],[8]. It estimates the main features of the image, after use a large regularization weight and gradually knows the image and filter details by slowly decreasing the regularization parameter The main drawback is it requires manual stopping and choose the final value of the regularization parameter the difference between the observed image and the blurred estimate is equals that of the noise is referred as discrepancy principle. Two popular criteria are generalized cross validation and the Lcurve [9] are developed and mainly applied to linear method also be used in non-linear methods. Most existing methods are requires regularization parameters to be empirically selected. for example, SURE-based approaches assumes full information about the degradation model but that are not suitable for BID. In the proposed criterion that can be used to adjust the regularization parameter and stopping iterative ID methods it is suitable for both NBID and BID problems. In the observed image the noise is spectrally white. The ratio is based on measures of spectral whiteness it represents the fitness of the current estimates to the degradation model.

\section{LITERATURE SURVEY}

After research issues have been extensively studied to give among the related work done. After many research had to be done image deblurring is categorized into two types are blind and non blind deblurring. To remove the (Iterative shrinkagethresholding algorithm) is solving for linear inverse problems in image processing. This method converge quite slowly so to move the new method FISTA (Fast Iterative ShrinkageThresholding Algorithm )preserves the calculations are simple compare with ISTA but global rate of convergence are significantly better for both theoretically and practically. Results for wavelet-based image deblurring exhibit the capacity of FISTA is faster than ISTA by several orders of 
magnitude [10]. The two-step IST (TwIST) algorithms exhibits much faster convergence rate than IST for illconditioned problems the main aim of this method keeping the good denoising performance of the IST scheme it able to handle ill-posed problems as efficiently. TwIST is to be converging much faster than the original IST in severely illconditioned problem to speed up and reach the two orders of magnitude in a typical deblurring problem. Problem of image restoration from missing samples is solved by new method MTWIST [11]. After many researches the result at the various intersections has been studied extensively. Previous methods in blind deblurring are very limited. They should not use the generic PSF. Most of them are based on a small number of parameters .from the above even a slight mismatch between the deblurring PSF and the blurring PSF strongly degrades the quality of the deblurred image. BID suffers from an understanding lack of data. Very few assumptions are made on blurring filter and original image To estimate of both deblurred image and blurring filter is made in progressive way first taking into account the main features of the image then proceed to smaller details. The results are obtained with synthetically blurred images are good even when the blur operator is ill-conditioned and the blurred image is noisy. This method yields improvements in real-life photographs with focus and motion blurs [12]. The algorithm works on natural images to different kinds of blurring, and de- blurs the image. In blind approach that minimal information about the blurring filter is needed to de-blur the image. This method applies to unconstrained blurs and can apply for both gray scale and color images. The algorithm uses the new image priors and increases the Signal to Noise ratio compare to the state of the art approaches [13].

\section{PROPOSED WORK}

In the proposed criteria for the convolution is applied to the input image to remove blur after to estimate the residual image (i.e., no blur is present in the image).finally got the output. The blind and non-blind ID as briefly discussed as follows: In the proposed method to do the both regularization and iterations for the unknown image or known image and stopping the iterations are based on fitness of the image is to be measured and compare to the degradation model to get residual image is $\mathrm{r}=\mathrm{y}-\hat{\mathrm{h}} * \hat{x}$ and degraded image is $\mathrm{y}=\mathrm{h} * \mathrm{x}+\mathrm{n}$ so here noise ' $n$ ' is assumed as the white.

\subsection{Image Deblurring/Deconvolution}

In image deblurring, the degraded image is modeled as follows as $y=h^{*} x+n$. Here ' $x$ ' is original image, ' $y$ ' is the degraded image, ' $n$ ' is the noise and ,'h' is the psf and * is blur from an image is called the deconvolution operation is originate the names of two methods the difference between the two methods are if the blur kernel or PSF is known is referred as non-blind deconvolution otherwise it is referred as blind deconvolution so to discuss the methods of each algorithms and it should be adopted in certain circumstances. A recent work manages ISTA the convolution. In NBID both the image and blur operator is to be assumed but in BID there is no assumption on image and blur operator. To find ' $x$ ' from ' $y$ ' and ' $h$ ' in NBID but in BID to find ' $x$ ' and ' $h$ ' from ' $y$ '

$$
\mathrm{C}_{\lambda}(\mathrm{x}, \mathrm{h})=1 / 2\|\mathrm{y}-\mathrm{h} * \mathrm{x}\| 22+\lambda \phi(\mathrm{x})
$$

Classical data fedility is the first term and results the noise ' $n$ ' is white and Gaussian $\phi(x)$ is the regularization function and information about the ' $x$ ' and ' $\lambda$ ' is the regularization parameter.

\subsection{Non-Blind Deblurring}

In NBID, to estimate the blur filter and also assume the ' $h$ ' is the point spread function is to be known and also minimize the cost function with respect to ' $\mathrm{x}$ ', choice is given to the regularization parameter ' $\lambda$ '. There are many methods for ID to minimize the cost function repeating a process and calculate to estimate the image at the iteration ' $t+1$ ' is a function of the previous estimate ' $x_{t}$ '. i.e., $x_{k+1}=f\left(x_{k}, y, h, \lambda\right.$ ).To estimate the regularization parameter ' $\lambda$ '. To repeat the process also need to stop the method so to consider the final results $\mathrm{r}=\mathrm{y}-\hat{\mathrm{h}} * \hat{x}$.

\subsection{Blind Deblurring}

In the proposed method to select the regularization parameter and final iteration so the whiteness starts to increase for certain peak after it starts to decrease so to stop the method for certain peak before the whiteness is decreases and measure the whiteness for starting point to certain peak.

\subsection{Measures of Whiteness}

First step of in our method is to make the regular of the residual image to make the mean is to be zero and the variance is unit so to denote the normalized residual is as

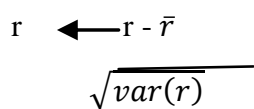

Where $\bar{r}$ and $\operatorname{var}(\mathrm{r})$ respectively .so the autocorrelation of the normalized residual ' $\mathrm{r}$ ' is estimated as

$\mathrm{R}_{\mathrm{rr}}(\mathrm{m}, \mathrm{n})=\mathrm{K} \sum_{i, j} r(i, j) r(i-m, j-n)$

Here where the summation is the residual image and ' $\mathrm{K}$ ' is the irrelevant constant. If $m=n=0$ then $\delta(m, n)=$ 1 , otherwise $\delta(m, n)=0$. So to measure of whiteness is based on the distance between the Rrr and a delta function. To consider a $(2 \mathrm{~L}+1) \mathrm{X}(2 \mathrm{~L}+1)$ so the proposed whiteness measure is simple the energy $R_{\mathrm{rr}}$ outside the origin

$$
M R(r)=-\sum_{\substack{(m, n)=(-L,-L) \\(m, n) \neq(0,0)}}^{L, L}(\operatorname{Rrr}(m, n))^{2}
$$

Where the minus sign is to make $\mathrm{M}_{\mathrm{R}}$ is larger for whiter residuals. The auto-covariance of large lags is smaller than that of small lags. The weighted version of measure is considered as

$$
\operatorname{MRW}(r)=-\sum_{\substack{(m, n)=(-L,-L) \\(m, n) \neq(0,0)}}^{L, L} W(m, n)(\operatorname{Rrr}(m, n))^{2}
$$

$\mathrm{W}(\mathrm{m}, \mathrm{n})$ is a weight of the matrix. The power spectral density of ' $\mathrm{r}$ ' is denote as $\mathrm{S}_{\mathrm{rr}}(\mathrm{w}, \mathrm{v})$ at $2 \mathrm{D}$ spatial frequency $(\mathrm{w}, \mathrm{v})$ $\mathrm{S}_{\mathrm{rr}}=\mathcal{F}(R r r)$

' $\mathcal{F}$ ' Represents the magnitude of the $2 \mathrm{D}$ discrete Fourier transform. The resulting measure is

$\mathrm{M}_{\mathrm{H}}(\mathrm{r})=-\sum_{w, v} \operatorname{Srr}(w, v) \log \operatorname{Srr}(w, v)$

Where $\mathrm{S}_{\mathrm{rr}}(\mathrm{w}, \mathrm{v})=\mathrm{S}_{\mathrm{rr}}(\mathrm{w}, \mathrm{v}) / \sum_{w^{\prime}, v^{\prime}} \operatorname{Srr}\left(w^{\prime}, v^{\prime}\right)$

To measure the whiteness of the local versions based on local auto-covariance estimates

$\mathrm{R}_{\mathrm{rr}}^{\mathrm{b}}(\mathrm{m}, \mathrm{n})=\sum_{i, j \in B b} r(i, j) r(i-m, j-n)$ 
Where ' $b$ ' is the indexes an image block and $B_{b}$ is the set of pixels in that block. So, to use the overlapping 9x9 blocks partially and separated horizontally and vertically by 5 pixels and fully contained in the image domain. So the residual is mean is to be zero and variance is unit. So this block partition the 3 local measure of whiteness $\mathrm{M}_{\mathrm{R}}^{\prime}, \mathrm{M}_{\mathrm{RW}}^{\prime}$ and $\mathrm{M}^{\prime} \mathrm{H}$ are obtained by calculating the corresponding local measures $M_{R}$, $\mathrm{M}_{\mathrm{RW}}$ and $\mathrm{MH}$ respectively at each block and average over all the blocks present in the image.

\section{INPUT OUTPUT SPECIFICATION AND DISCUSSION}

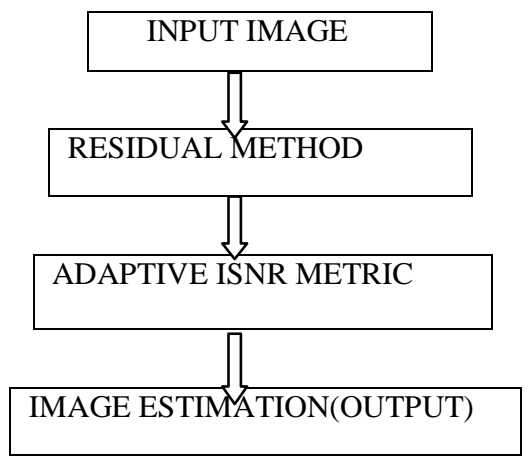

Fig1: Adaptive method for whiteness measurement

From the above fig1 can be explained with examples as follows:

\subsection{Input Images}

In single frame scenarios

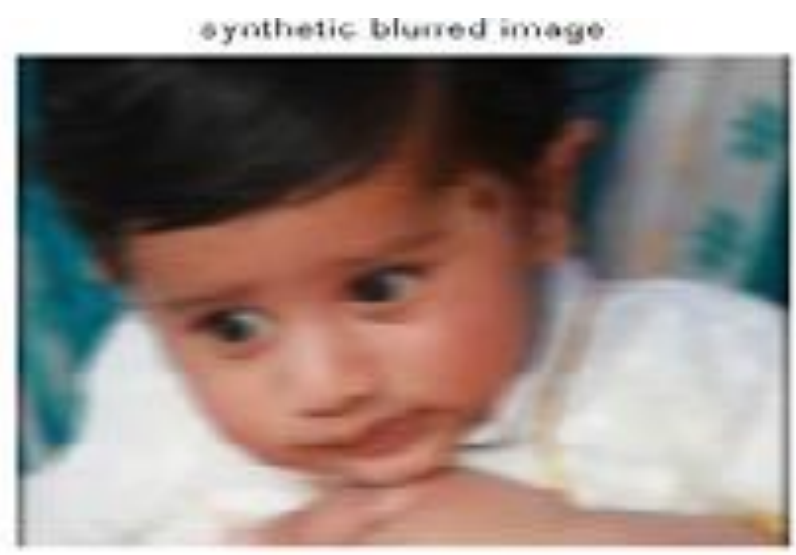

Fig(2) synthetic blurred image blur with noise added image

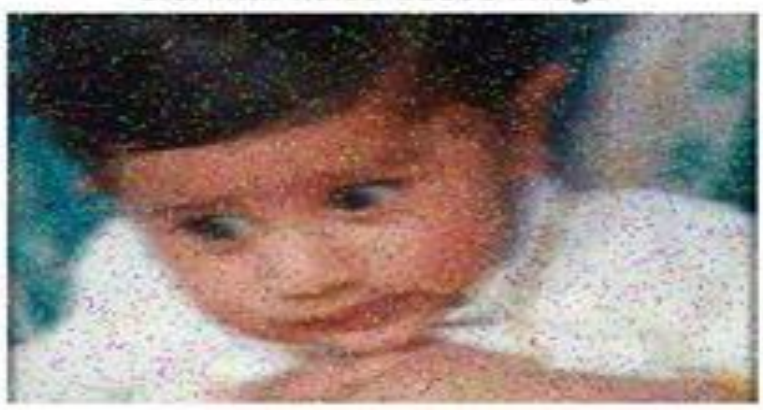

Fig(3) blur with noise added image

From the above fig (2) \& (3) by using camera to capture the image synthetically some amount of blur and noise is added to the image.

\subsection{Residual Method}

The images below can be obtained during the working of residual method.
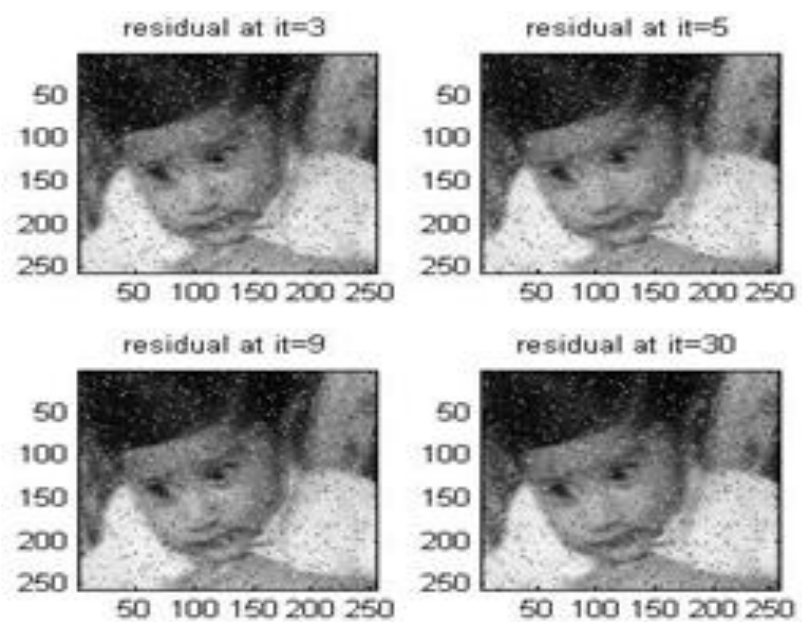

Fig (4) working of residual method with different iterations

From the above fig(4) after capturing the image first to do the regularization means arrange the pixels in normalized form after to do the iterations means the same process is repeated starts from iteration 1 to and so on up to got the blur removed image called residual image.

\subsection{Adaptive ISNR Metric}
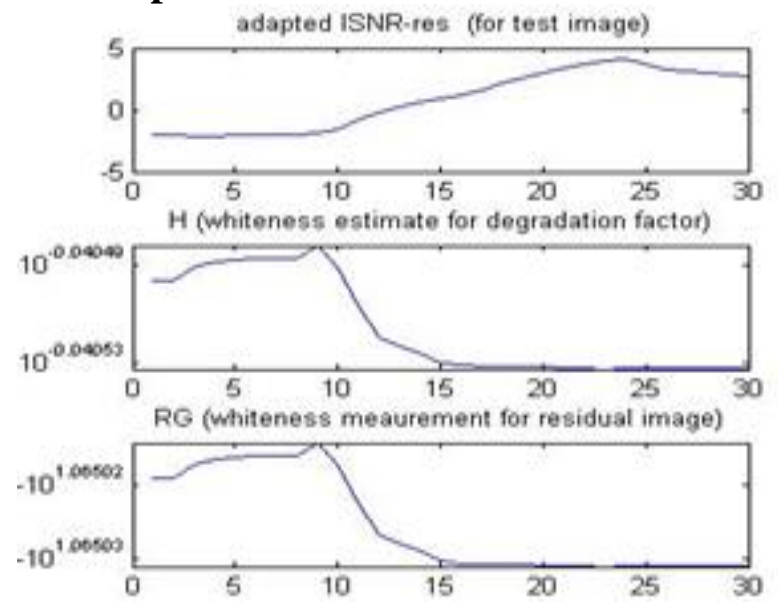

Fig (5): plot graph from Adaptive ISNR method

From the above fig (5) plot the Adaptive ISNR for output images is the blurred image and ' $R G$ ' is the whiteness measurement for the residual image. If the ISNR value is decreases the noise is removed.

Table 1

\begin{tabular}{|l|l|l|l|l|}
\hline $\begin{array}{l}\text { Salt\& } \\
\text { pepper } \\
\text { noise }\end{array}$ & R RG H & R RGBHB & $\begin{array}{l}\text { Best } \\
\text { ISNR }\end{array}$ & $\begin{array}{l}\text { Best } \\
\text { ISNR }\end{array}$ \\
\hline 0.01 & 292429 & 232323 & 23 & 24 \\
\hline 0.02 & 192319 & 232323 & 23 & 24 \\
\hline
\end{tabular}




\begin{tabular}{|l|l|l|l|l|}
\hline 0.03 & 202321 & 232323 & 23 & 24 \\
\hline 0.04 & 192420 & 232323 & 23 & 24 \\
\hline 0.05 & 192319 & 232328 & 23 & 24 \\
\hline 0.06 & 192219 & 232329 & 23 & 24 \\
\hline 0.07 & 182418 & 252427 & 23 & 24 \\
\hline 0.08 & 182318 & 302327 & 23 & 24 \\
\hline 0.09 & 192419 & 282229 & 23 & 24 \\
\hline 0.1 & 181918 & 262820 & 23 & 24 \\
\hline 0.2 & 161716 & 303030 & 23 & 24 \\
\hline 0.3 & 111111 & 191919 & 23 & 24 \\
\hline 0.4 & 101010 & 112923 & 23 & 24 \\
\hline 0.5 & 999 & 9119 & 23 & 24 \\
\hline
\end{tabular}

In multiframe scenarios

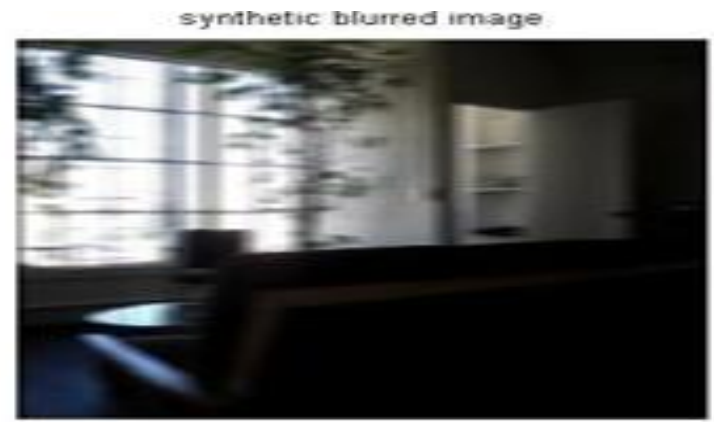

Fig (7) synthetic blurred image

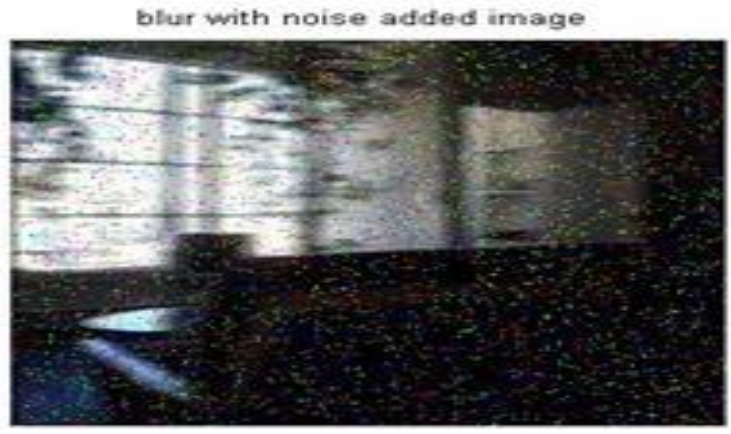

Fig (8) blur with noise added image

From the above fig (7) \& (8) by using camera to capture the image synthetically some amount of blur and noise is added to the image.

the algorithm was stopped based on each of the criteria.

\subsection{Output Images}

The images below can be obtained after the working of residual method and Adaptive ISNR method

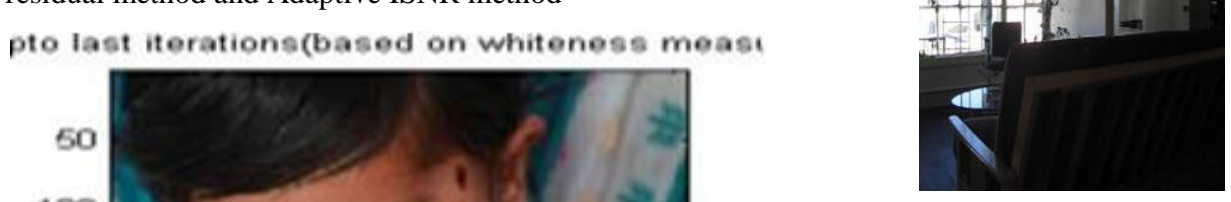

Fig (9)

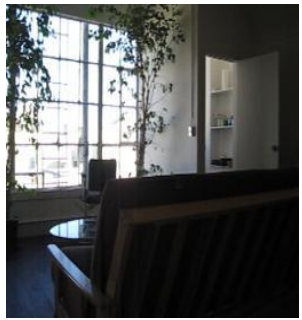

Fig (11)

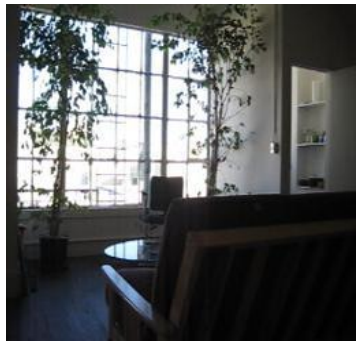

Fig (13)

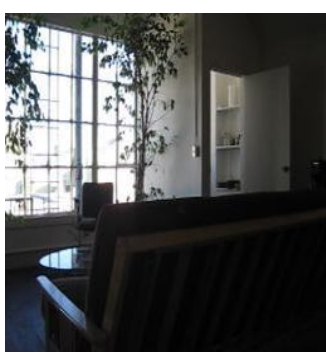

fig (10)

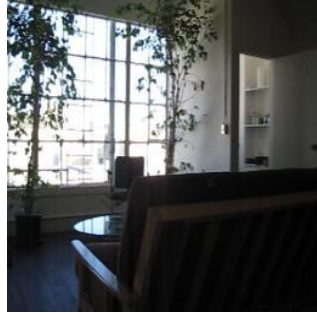

fig (12)

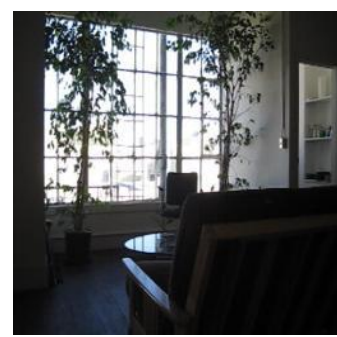

fig (14) 
From the above figures $9,10,11,12,13,14$ are the images capture from the movie so now select the one image and do the residual and Adaptive ISNR method.

The images below can be obtained during the working of residual method.

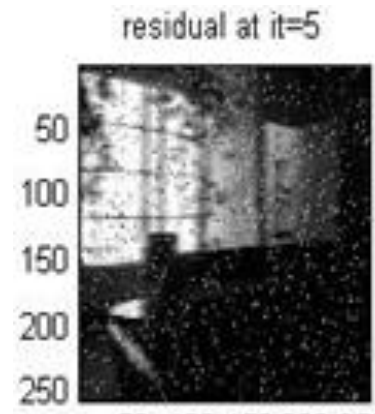

50100150200250

residual at it $=10$

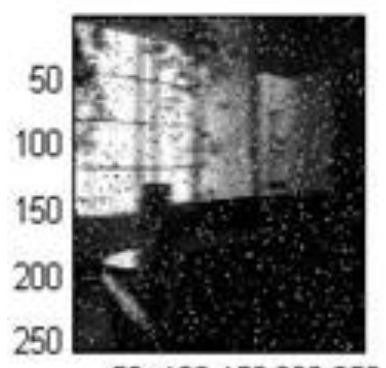

50100150200250

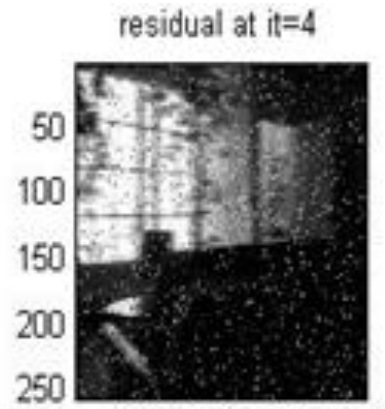

50100150200250

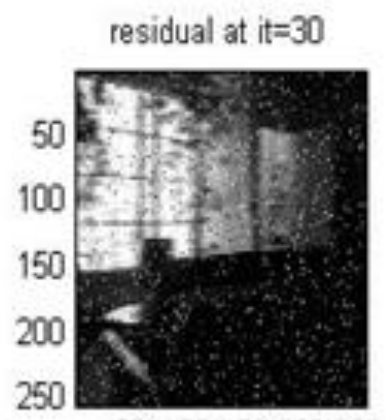

50100150200250
Fig (15) working of residual method with different iterations

The procedure is same as like single frame scenarios.
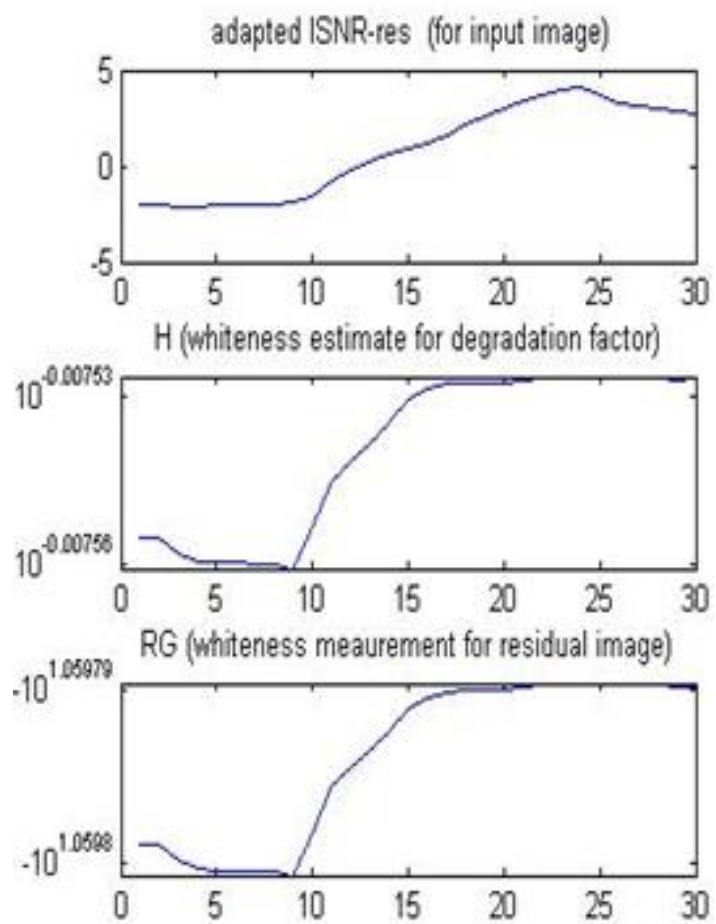

Fig (16):plot graph from Adaptive ISNR method
Table 2

\begin{tabular}{|c|c|c|c|c|}
\hline $\begin{array}{l}\text { Salt\& } \\
\text { pepper } \\
\text { noise }\end{array}$ & R RG H & R RGBHB & $\begin{array}{l}\text { Best } \\
\text { ISNR }\end{array}$ & $\begin{array}{l}\text { Best } \\
\text { ISNR }\end{array}$ \\
\hline 0.01 & 262626 & 101010 & 23 & 24 \\
\hline 0.02 & 262626 & 101010 & 23 & 24 \\
\hline 0.03 & 282828 & 10109 & 23 & 24 \\
\hline 0.04 & 242424 & 101010 & 23 & 24 \\
\hline 0.05 & 282828 & 101010 & 23 & 24 \\
\hline 0.06 & 262626 & 9910 & 23 & 24 \\
\hline 0.07 & 252525 & 999 & 23 & 24 \\
\hline 0.08 & 262628 & 9910 & 23 & 24 \\
\hline 0.09 & 222222 & 999 & 23 & 24 \\
\hline 0.1 & 232323 & 999 & 23 & 24 \\
\hline 0.2 & 282828 & 9910 & 23 & 24 \\
\hline 0.3 & 232323 & 999 & 23 & 24 \\
\hline 0.4 & 282828 & 282827 & 23 & 24 \\
\hline 0.5 & 282328 & 151515 & 23 & 24 \\
\hline
\end{tabular}

From the above table- 1 the numbers are the iterations at which the algorithm was stopped based on each of the criteria.

The images below can be obtained after the working of residual method and Adaptive ISNR method. 


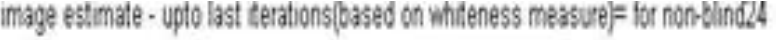

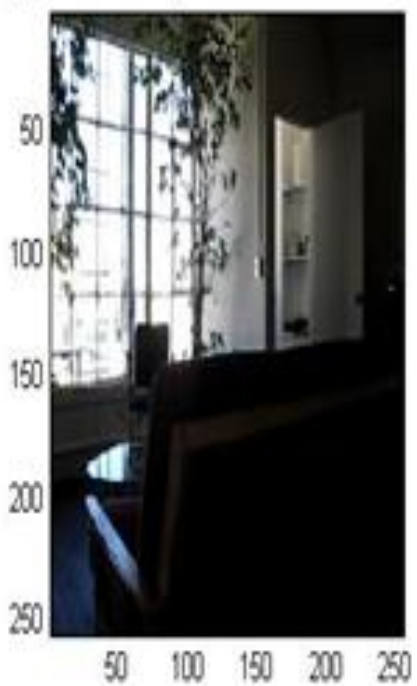

image estimate - upto last terations(based on whiteness measure) for blind mathod=10

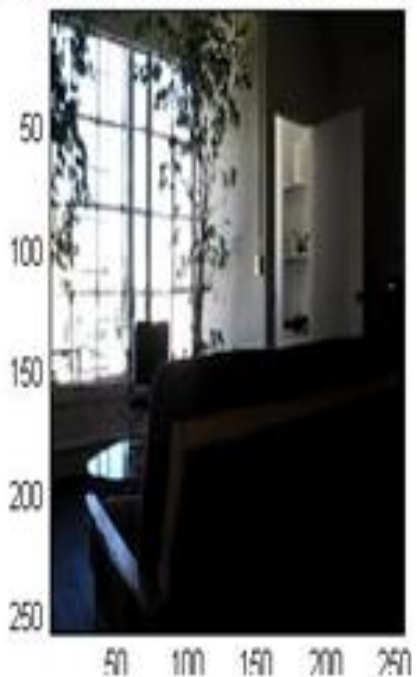

Fig (17): Images are obtained for non-Blind and Blind method

\section{CONCLUSION}

From the above analysis to conclude that it is accurate but not active to different types of blurs and lighting problems to make the deblurring is difficult. so in the previous methods can be used to select the regularization parameter then to stop iterative blind and non-blind deconvolution algorithm. But in proposal is based on whiteness of the residual image is measured. Proposed method is to be handled both constrained and unconstrained domain images. It makes weak assumptions about the blurring filter and focus on the main edges of the image and gradually takes details into account .It works on single-frame scenarios both monochrome and color images then synthetic and real-life degradations. Ongoing research aims at a significant speed up (by using the ADMM) and ability to handle the unknown boundaries and also developing adequate stopping criteria for the inner ADMM algorithms as well as for the outer iterations.

\section{ACKNOWLEDGEMENT}

We are very grateful to the editor and the reviewers for their valuable comments and suggestions to help improving this work

\section{REFERENCES}

[1] J. M. Bivouacs-Dias and M. A. T. Figueiredo, "A newTwIST: Two-step iterative shrinkage/thresholding algorithms for image restoration," IEEE Trans. on Image Processing, vol. 16, pp. 2992 - 3004, 2007.

[2] G. Chantas, N. Galatsanos, A. Likas, and M. Saunders, "Variational Bayesian image restoration based on a product of t-distributions image prior," IEEE Trans. Image Processing, vol. 17, pp. 1795-805, 2008.

[3] M. Elad, M. A. T. Figueiredo, and Y. Ma, "On the role of sparse and redundant representations in image processing," Proceedings of the IEEE, vol. 98, pp. 972 982,2010

[4] J.-L. Starck, F. Murtagh, and J. Fadili, Sparse Image and Signal Processing: Wavelets, Curvelets, Morphological Diversity. CambridgeUniversity Press, 2010.

[5] A. S. Carasso, "The APEX method in image sharpening and the use of low exponent L'evy stable laws," SIAM Journal of Applied Mathematics, vol. 63, pp. 593-618, 2003

[6] M. S. Chang, S. W. Yun, and P. Park, "PSF search algorithm for dual-exposure type blurred image," Int. Journal of Applied Science, Engineering and Technology, vol. 4, 2007

[7] B. Amizic, S. D. Babacan, R. Molina, and A. K. Katsaggelos, "Sparse Bayesian blind image deconvolution with parameter estimation," in European Signal Processing Conference, 2010

[8] S. D. Babacan, R. Molina, and A. K. Katsaggelos, "Variational Bayesian blind deconvolution using a total variation prior," IEEE Trans. Image Processing, vol. 18, pp. 12-26, 2009.

[9] G. Golub, M. Heath, and G. Wahba, "Generalized crossvalidation as amethod for choosing a good ridge parameter," Technometrics, vol. 21, pp. 215-223, 1979

[10] Spatial operations, http://zernike.u winnipeg.ca/ s_liao/Courses/7205/Week03

[11 ] J. Y. im, L. S. Kim, S. H Hwang, "An advanced Contrast Enhancement Using Partially Overlapped Sub Block Histogram Equalization",IEEE Transactions on Circuits and Systems for Videc Technology, Vol. 11, No. 4, pp.475-484,2001

[12] M. A. T. Figueiredo and R. D. Nowak, "An EM algorithm for waveletbased image restoration" IEEE Trans. Image Processing, vol. 12, no. 8, pp. 906-916, 2

[13] M. S. C. Almeida and L. B. Almeida, "Blind deblurring of natural images," in IEEE Int. Conf. Acoustics, Speech, and Signal Processing - ICASSP, 2008, pp. 1261-1264. 